\title{
An Unusual Case of Hypercalcaemia whilst Severely Hypomagnesaemic
}

\author{
Fatima Alkaabi ${ }^{1}$, Ali Naqvi ${ }^{1}$, Chong Lim ${ }^{1}$, Ali Abbara ${ }^{2}$, Alexander N Comninos ${ }^{1,2}$, Jeremy Cox ${ }^{1}$ \\ ${ }^{1}$ Department of Endocrinology, St. Mary's Hospital, Imperial College Healthcare NHS Trust, UK \\ Section of Endocrinology \& Investigative Medicine, Imperial College London, UK
}

\begin{abstract}
BACKGROUND
Hypomagnesaemia can be induced by gastrointestinal or renal loss. It is often associated with hypokalaemia (due to urinary potassium wasting) and hypocalcaemia (due to lower parathyroid hormone secretion and end-organ resistance to its effect). Moreover, patient with hypercalcemia can develop hypomagnesaemia because the calcium and magnesium functionally compete for transport in the thick ascending limb of the loop of Henle.

Hypomagnesaemia with hypocalcaemia, has been described in case reports with the chronic use of proton pump inhibitors (PPI) for typically more than 1 year. The association between PPI use and magnesium levels varies by whether patients concurrently use diuretics. In patients taking diuretics, concurrent use of PPIs was associated with lower adjusted serum magnesium compared to patient not taking diuretics (1-4).

By contrast the occurrence of hypercalcaemia in the context of hypomagnesaemia is unusual. Here, we present a case from the
\end{abstract} metabolic bone clinic

\section{CASE}

History \& Examination

A 68-year-old woman presented with a several month history of nausea, confusion and generalised weakness. In addition, she reported decreased appetite with significant weight loss. She did not report any convulsions, abdominal pain, palpitations, or diarrhoea.

Significant past medical history included well-controlled T2DM, hypertension, vitamin D deficiency and GI reflux disease. Importantly, she had no history of renal disease.

Relevant medications included calcium+vitamin D supplements, indapamide and omeprazole. She had no significant family history and did not drink alcohol.

Examination was unremarkable, with no tetany or abnormal reflexes. Neck examination was normal.

\section{Investigations}

Blood tests revealed an undetectable magnesium $(<0.25 \mathrm{mmol} / \mathrm{L}$, NR 0.7 $1.0 \mathrm{mmol} / \mathrm{L}$ ), high adjusted calcium of $3.15 \mathrm{mmol} / \mathrm{L}$ (NR $2.2-2.6 \mathrm{mmol} / \mathrm{L}$ ), with a normal phosphate. PTH was in the mid normal range at $3.9 \mathrm{pmol} / \mathrm{L}$ (NR 1.1-6.8pmol/L), and renal function was normal.

Electrocardiogram demonstrated Right-Bundle-Branch-Block with no features of hypercalcaemia.

\section{Management}

She was managed as follows:

Multiple intravenous magnesium infusions

Indapamide stopped

Calcium+vitamin D stopped

Omeprazole stopped

This resulted in normalisation of magnesium and calcium levels with concomitant relief of her symptoms. (Figures 1 \& 2 )

\section{Subsequent care}

Neck ultrasound did not identify a parathyroid adenoma.

Four weeks post-discharge, her adjusted calcium was at the top of the normal range $(2.59 \mathrm{mmol} / \mathrm{L})$ with PTH $5.9 \mathrm{pmol} / \mathrm{L}$, vitamin D $66 \mathrm{nmol} / \mathrm{L}$ and magnesium $0.79 \mathrm{mmol} / \mathrm{L}$.

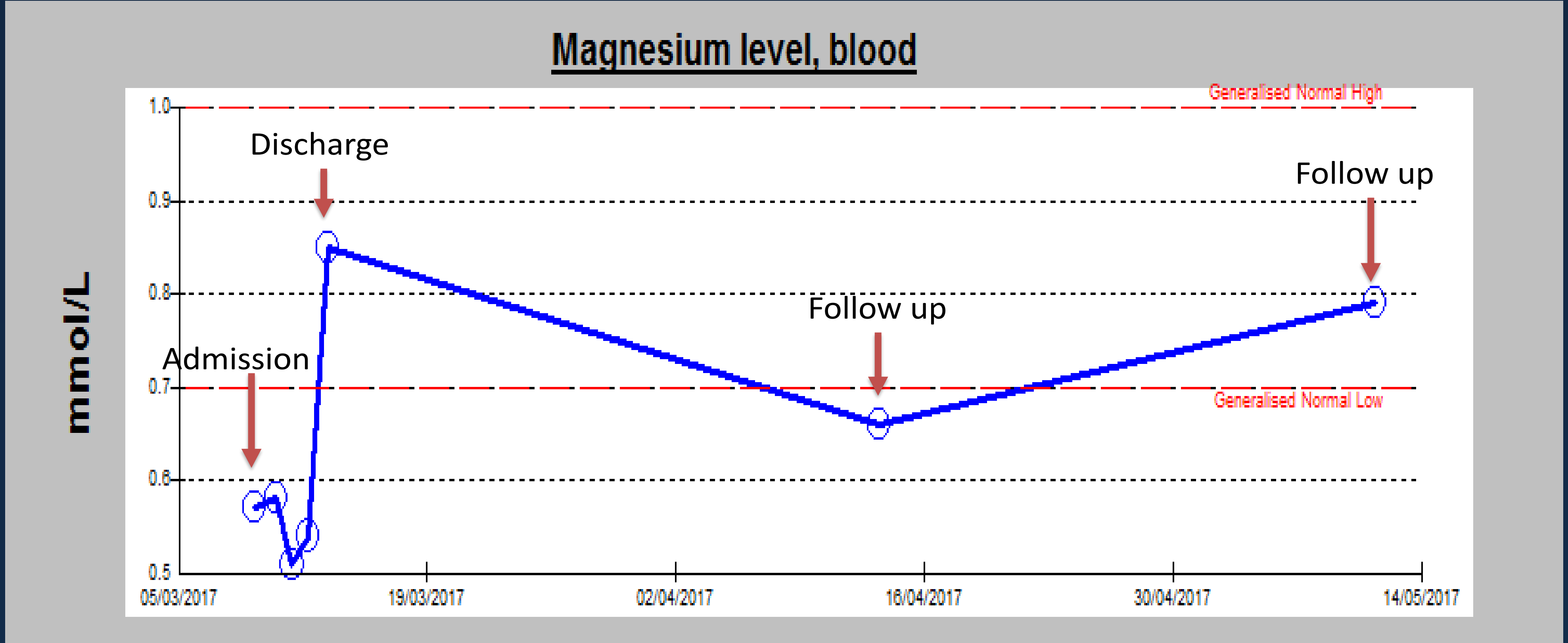

Figure 1

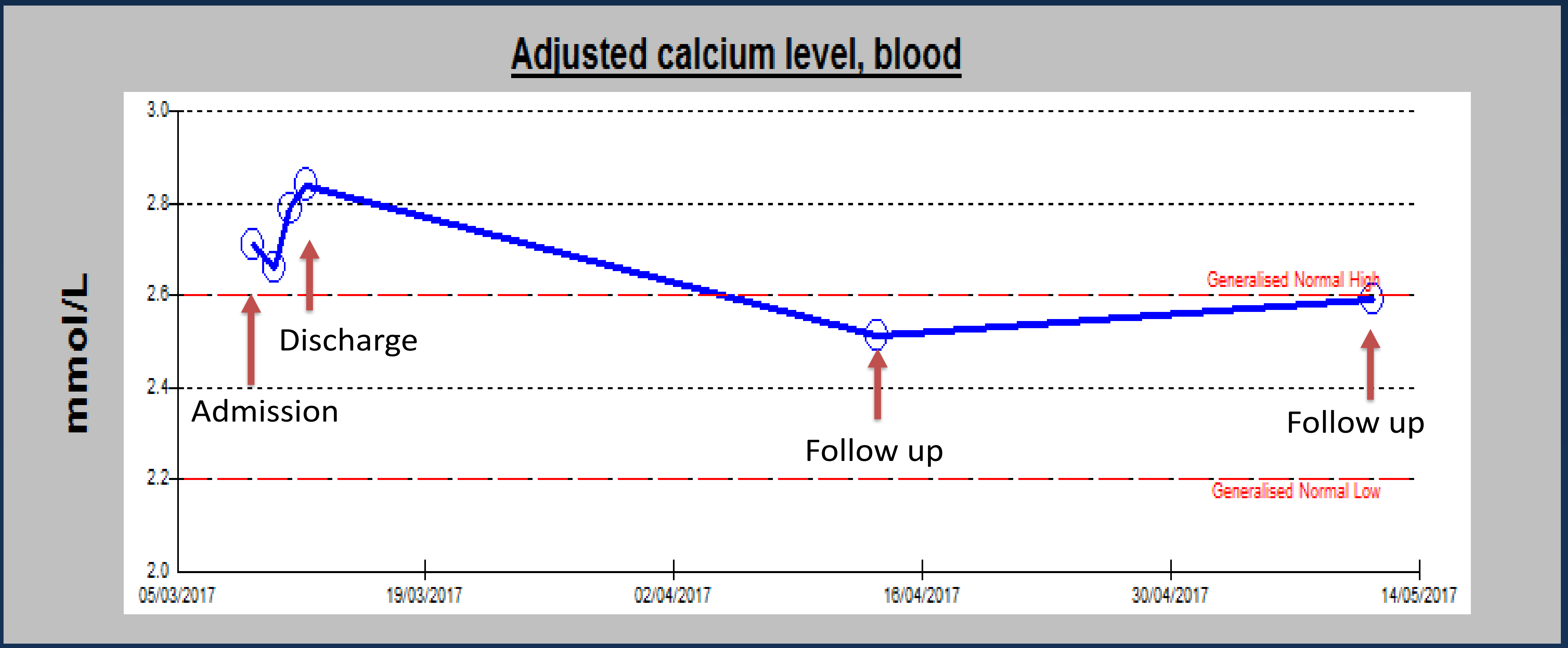

Figure2

\section{DISCUSSION}

This patients hypercalcaemia was either purely indapamide + calcium supplementation induced or could represent the unmasking of mild primary hyperparathyroidism. Omeprazole use markedly reduced intestinal magnesium absorption; the hypercalcaemia prevented renal compensation for this hypomagnesaemia by reducing renal magnesium reabsorption in the thick ascending limb of the loop of Henle. This was further compounded by the thiazide reducing distal tubule magnesium reabsorption.(Figure 3)

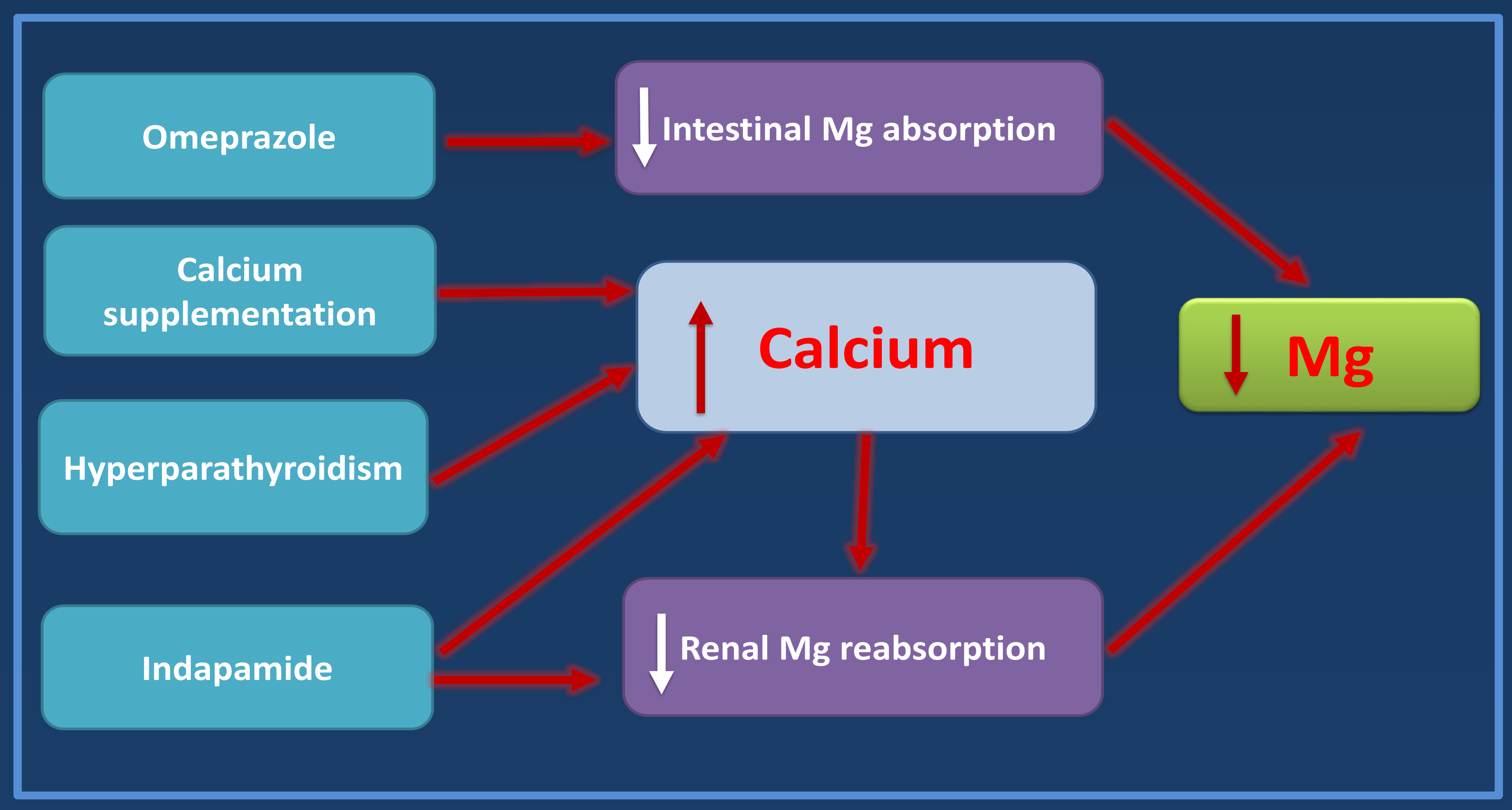

Figure3

This case highlights the need to be extra-vigilant in patients on concomitant PPIs and diuretics as there is a risk of severe hypomagnesemia. Furthermore, this case may provide an example of the unmasking of hyperparathyroidism by the combination of thiazides and calcium supplements.

\section{REFERENCES}

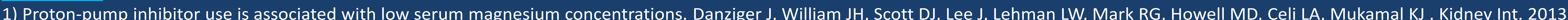
Apr;83(4):692-9. Epub 2013 Jan 16.

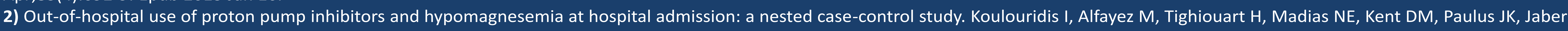
BL. Am J Kidney Dis. 2013;62(4):730. Epub 2013 May 10.

3) The association between the use of proton pump inhibitors and the risk of hypomagnesemia: a systematic review and meta-analysis. Park CH, Kim EH, Roh YH, Kim HY, Lee SK . PLoS One. 2014;9(11):e112558. Epub 2014 Nov 13.

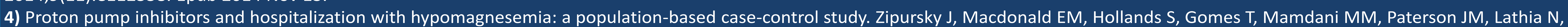
Juurlink DN . PLoS Med. 2014;11(9):e1001736. Epub 2014 Sep 30 